CERN-PPE/93-110

5 July 1993

\title{
Search for the Standard Model Higgs Boson
}

\author{
The ALEPH Collaboration*)
}

\begin{abstract}
$\underline{\text { Abstract }}$
Using a data sample corresponding to about $1,233,000$ hadronic Z decays collected by the ALEPH experiment at LEP, the reaction $\mathrm{e}^{+} \mathrm{e}^{-} \rightarrow \mathrm{HZ}^{*}$ has been used to search for the standard model Higgs boson, in association with missing energy when $\mathrm{Z}^{*} \rightarrow \nu \bar{\nu}$, or with a pair of energetic leptons when $\mathrm{Z}^{*} \rightarrow \mathrm{e}^{+} \mathrm{e}^{-}$or $\mu^{+} \mu^{-}$. No signal was found and, at the $95 \%$ confidence level, $m_{\mathrm{H}}$ exceeds $58.4 \mathrm{GeV} / c^{2}$.
\end{abstract}

(Submitted to Physics Letters B)

*) See next pages for the list of authors. 



\section{The ALEPH Collaboration}

D. Buskulic, I. De Bonis, D. Decamp, P. Ghez, C. Goy, J.-P. Lees, M.-N. Minard, B. Pietrzyk Laboratoire de Physique des Particules (LAPP), IN ${ }^{2} P^{3}$-CNRS, 74019 Annecy-le-Vieux Cedex, France

F. Ariztizabal, P. Comas, J.M. Crespo, M. Delfino, I. Efthymiopoulos, E. Fernandez, M. Fernandez-Bosman,

V. Gaitan, Ll. Garrido, T. Mattison, A. Pacheco, C. Padilla, A. Pascual Institut de Fisica d'Altes Energies, Universitat Autonoma de Barcelona, 08193 Bellaterra (Barcelona), $\operatorname{Spain}^{7}$

D. Creanza, M. de Palma, A. Farilla, G. Iaselli, G. Maggi, S. Natali, S. Nuzzo, M. Quattromini, A. Ranieri, G. Raso, F. Romano, F. Ruggieri, G. Selvaggi, L. Silvestris, P. Tempesta, G. Zito INFN Sezione di Bari e Dipartimento di Fisica dell' Università, 70126 Bari, Italy

Y. Chai, H. Hu, D. Huang, X. Huang, J. Lin, T. Wang, Y. Xie, D. Xu, R. Xu, J. Zhang, L. Zhang, W. Zhao Institute of High-Energy Physics, Academia Sinica, Beijing, The People's Republic of China ${ }^{8}$

E. Blucher, ${ }^{22}$ G. Bonvicini, J. Boudreau, D. Casper, H. Drevermann, R.W. Forty, G. Ganis, C. Gay, R. Hagelberg, J. Harvey, J. Hilgart, ${ }^{31}$ R. Jacobsen, B. Jost, J. Knobloch, I. Lehraus, T. Lohse, ${ }^{27}$ M. Maggi, C. Markou, M. Martinez, P. Mato, H. Meinhard, A. Minten, A. Miotto, R. Miquel, H.-G. Moser, P. Palazzi, J.R. Pater, J.A. Perlas, J.-F. Pusztaszeri, F. Ranjard, G. Redlinger, ${ }^{23}$ L. Rolandi, J. Rothberg, ${ }^{2}$ T. Ruan, M. Saich, D. Schlatter, M. Schmelling, F. Sefkow, ${ }^{6}$ W. Tejessy, I.R. Tomalin, R. Veenhof, H. Wachsmuth, S. Wasserbaech, ${ }^{2}$ W. Wiedenmann, T. Wildish, W. Witzeling, J. Wotschack European Laboratory for Particle Physics (CERN), 1211 Geneva 23, Switzerland

Z. Ajaltouni, F. Badaud, M. Bardadin-Otwinowska, R. El Fellous, A. Falvard, P. Gay, C. Guicheney, P. Henrard, J. Jousset, B. Michel, J-C. Montret, D. Pallin, P. Perret, F. Podlyski, J. Proriol, F. Prulhière, F. Saadi Laboratoire de Physique Corpusculaire, Université Blaise Pascal, IN ${ }^{2} P^{3}$-CNRS, Clermont-Ferrand, 63177 Aubière, France

T. Fearnley, J.B. Hansen, J.D. Hansen, J.R. Hansen, ${ }^{1}$ P.H. Hansen, R. Møllerud, B.S. Nilsson ${ }^{1}$ Niels Bohr Institute, 2100 Copenhagen, Denmark ${ }^{9}$

A. Kyriakis, E. Simopoulou, I. Siotis, A. Vayaki, K. Zachariadou Nuclear Research Center Demokritos (NRCD), Athens, Greece

J. Badier, A. Blondel, G. Bonneaud, J.C. Brient, G. Fouque, S. Orteu, A. Rougé, M. Rumpf, R. Tanaka, M. Verderi, H. Videau Laboratoire de Physique Nucléaire et des Hautes Energies, Ecole Polytechnique, IN ${ }^{2} P^{3}{ }_{-}$CNRS, 91128 Palaiseau Cedex, France

D.J. Candlin, M.I. Parsons, E. Veitch Department of Physics, University of Edinburgh, Edinburgh EH9 3JZ, United Kingdom ${ }^{10}$

E. Focardi, L. Moneta, G. Parrini Dipartimento di Fisica, Università di Firenze, INFN Sezione di Firenze, 50125 Firenze, Italy

M. Corden, C. Georgiopoulos, M. Ikeda, D. Levinthal ${ }^{15}$ Supercomputer Computations Research Institute and Dept. of Physics, Florida State University, Tallahassee, FL 32306, USA $12,13,14$

A. Antonelli, R. Baldini, G. Bencivenni, G. Bologna, ${ }^{4}$ F. Bossi, P. Campana, G. Capon, F. Cerutti, V. Chiarella, B. D’Ettorre-Piazzoli, ${ }^{24}$ G. Felici, P. Laurelli, G. Mannocchi, ${ }^{5}$ F. Murtas, G.P. Murtas, L. Passalacqua, M. PepeAltarelli, P. Picchi ${ }^{4}$ Laboratori Nazionali dell'INFN (LNF-INFN), 00044 Frascati, Italy 
P. Colrain, I. ten Have, J.G. Lynch, W. Maitland, W.T. Morton, C. Raine, P. Reeves, J.M. Scarr, K. Smith, M.G. Smith, A.S. Thompson, R.M. Turnbull

Department of Physics and Astronomy, University of Glasgow, Glasgow G12 8QQ, United Kingdom ${ }^{10}$

B. Brandl, O. Braun, C. Geweniger, P. Hanke, V. Hepp, E.E. Kluge, Y. Maumary, A. Putzer, B. Rensch, A. Stahl, K. Tittel, M. Wunsch

Institut für Hochenergiephysik, Universität Heidelberg, 6900 Heidelberg, Fed. Rep. of Germany ${ }^{16}$

R. Beuselinck, D.M. Binnie, W. Cameron, M. Cattaneo, D.J. Colling, P.J. Dornan, A.M. Greene, J.F. Hassard, N.M. Lieske, ${ }^{29}$ A. Moutoussi, J. Nash, S. Patton, D.G. Payne, M.J. Phillips, G. San Martin, J.K. Sedgbeer, A.G. Wright

Department of Physics, Imperial College, London SW7 2BZ, United Kingdom ${ }^{10}$

P. Girtler, D. Kuhn, G. Rudolph, R. Vogl

Institut für Experimentalphysik, Universität Innsbruck, 6020 Innsbruck, Austria ${ }^{18}$

C.K. Bowdery, T.J. Brodbeck, A.J. Finch, F. Foster, G. Hughes, D. Jackson, N.R. Keemer, M. Nuttall, A. Patel, T. Sloan, S.W. Snow, E.P. Whelan

Department of Physics, University of Lancaster, Lancaster LA1 4YB, United Kingdom ${ }^{10}$

K. Kleinknecht, J. Raab, B. Renk, H.-G. Sander, H. Schmidt, F. Steeg, S.M. Walther, R. Wanke, B. Wolf Institut für Physik, Universität Mainz, 6500 Mainz, Fed. Rep. of Germany ${ }^{16}$

A.M. Bencheikh, C. Benchouk, A. Bonissent, J. Carr, P. Coyle, J. Drinkard, ${ }^{3}$ F. Etienne, D. Nicod, S. Papalexiou, P. Payre, L. Roos, D. Rousseau, P. Schwemling, M. Talby

Centre de Physique des Particules, Faculté des Sciences de Luminy, IN ${ }^{2} P^{3}$-CNRS, 13288 Marseille,

France

S. Adlung, R. Assmann, C. Bauer, W. Blum, D. Brown, P. Cattaneo, ${ }^{26}$ B. Dehning, H. Dietl, F. Dydak, ${ }^{21}$ M. Frank, A.W. Halley, K. Jakobs, J. Lauber, G. Lütjens, G. Lutz, W. Männer, R. Richter, J. Schröder, A.S. Schwarz, R. Settles, H. Seywerd, U. Stierlin, U. Stiegler, R. St. Denis, G. Wolf Max-Planck-Institut für Physik, Werner-Heisenberg-Institut, 8000 München, Fed. Rep. of Germany ${ }^{16}$

R. Alemany, J. Boucrot, ${ }^{1}$ O. Callot, A. Cordier, M. Davier, L. Duflot, J.-F. Grivaz, Ph. Heusse, D.E. Jaffe, P. Janot, D.W. Kim ${ }^{19}$ F. Le Diberder, J. Lefrançois, A.-M. Lutz, M.-H. Schune, J.-J. Veillet, I. Videau, Z. Zhang Laboratoire de l'Accélérateur Linéaire, Université de Paris-Sud, IN ${ }^{2} P^{3}$ CNRS, 91405 Orsay Cedex, France

D. Abbaneo, G. Bagliesi, G. Batignani, U. Bottigli, C. Bozzi, G. Calderini, M. Carpinelli, M.A. Ciocci, R. Dell'Orso, I. Ferrante, F. Fidecaro, L. Foà, F. Forti, A. Giassi, M.A. Giorgi, A. Gregorio, F. Ligabue, A. Lusiani, E.B. Mannelli, P.S. Marrocchesi, A. Messineo, F. Palla, G. Rizzo, G. Sanguinetti, P. Spagnolo, J. Steinberger, R. Tenchini, G. Tonelli, ${ }^{32}$ G. Triggiani, C. Vannini, A. Venturi, P.G. Verdini, J. Walsh

Dipartimento di Fisica dell'Università, INFN Sezione di Pisa, e Scuola Normale Superiore, 56010 Pisa, Italy

A.P. Betteridge, Y. Gao, M.G. Green, P.V. March, Ll.M. Mir, T. Medcalf, I.S. Quazi, J.A. Strong, L.R. West Department of Physics, Royal Holloway \& Bedford New College, University of London, Surrey TW20 OEX, United Kingdom ${ }^{10}$

D.R. Botterill, R.W. Clifft, T.R. Edgecock, S. Haywood, P.R. Norton, J.C. Thompson Particle Physics Dept., Rutherford Appleton Laboratory, Chilton, Didcot, Oxon OX11 OQX, United King dom ${ }^{10}$

B. Bloch-Devaux, P. Colas, H. Duarte, S. Emery, W. Kozanecki, E. Lançon, M.C. Lemaire, E. Locci, B. Marx, P. Perez, J. Rander, J.-F. Renardy, A. Rosowsky, A. Roussarie, J.-P. Schuller, J. Schwindling, D. Si Mohand, B. Vallage

Service de Physique des Particules, DAPNIA, CE-Saclay, 91191 Gif-sur-Yvette Cedex, France ${ }^{17}$ 
R.P. Johnson, A.M. Litke, G. Taylor, J. Wear

Institute for Particle Physics, University of California at Santa Cruz, Santa Cruz, CA 95064, USA ${ }^{25}$

J.G. Ashman, W. Babbage, C.N. Booth, C. Buttar, S. Cartwright, F. Combley, I. Dawson, L.F. Thompson

Department of Physics, University of Sheffield, Sheffield S3 $7 R H$, United Kingdom ${ }^{10}$

E. Barberio, A. Böhrer, S. Brandt, G. Cowan, C. Grupen, G. Lutters, F. Rivera, ${ }^{30}$ U. Schäfer, L. Smolik

Fachbereich Physik, Universität Siegen, 5900 Siegen, Fed. Rep. of Germany ${ }^{16}$

L. Bosisio, R. Della Marina, G. Giannini, B. Gobbo, F. Ragusa ${ }^{20}$

Dipartimento di Fisica, Università di Trieste e INFN Sezione di Trieste, 34127 Trieste, Italy

L. Bellantoni, W. Chen, J.S. Conway, ${ }^{28}$ Z. Feng, D.P.S. Ferguson, Y.S. Gao, J. Grahl, J.L. Harton, O.J. Hayes III, J.M. Nachtman, Y.B. Pan, Y. Saadi, M. Schmitt, I. Scott, V. Sharma, Z.H. Shi, J.D. Turk, A.M. Walsh, F.V. Weber, Sau Lan Wu, X. Wu, M. Zheng, G. Zobernig

Department of Physics, University of Wisconsin, Madison, WI 53706, USA ${ }^{11}$

\footnotetext{
${ }^{1}$ Now at CERN, PPE Division, 1211 Geneva 23 , Switzerland.

${ }^{2}$ Permanent address: University of Washington, Seattle, WA 98195, USA.

${ }^{3}$ Now at University of California, Irvine, CA 92717, USA.

${ }^{4}$ Also Istituto di Fisica Generale, Università di Torino, Torino, Italy.

${ }^{5}$ Also Istituto di Cosmo-Geofisica del C.N.R., Torino, Italy.

${ }^{6}$ Now at DESY, Hamburg, Germany.

${ }^{7}$ Supported by CICYT, Spain.

${ }^{8}$ Supported by the National Science Foundation of China.

${ }^{9}$ Supported by the Danish Natural Science Research Council.

${ }^{10}$ Supported by the UK Science and Engineering Research Council.

${ }^{11}$ Supported by the US Department of Energy, contract DE-AC02-76ER00881.

${ }^{12}$ Supported by the US Department of Energy, contract DE-FG05-87ER40319.

${ }^{13}$ Supported by the NSF, contract PHY-8451274.

${ }^{14}$ Supported by the US Department of Energy, contract DE-FC05-85ER250000.

${ }^{15}$ Supported by SLOAN fellowship, contract BR 2703.

${ }^{16}$ Supported by the Bundesministerium für Forschung und Technologie, Fed. Rep. of Germany.

${ }^{17}$ Supported by the Direction des Sciences de la Matière, C.E.A.

${ }^{18}$ Supported by Fonds zur Förderung der wissenschaftlichen Forschung, Austria.

${ }^{19}$ Supported by the Korean Science and Engineering Foundation and Ministry of Education.

${ }^{20}$ Now at Dipartimento di Fisica, Università di Milano, Milano, Italy.

${ }^{21}$ Also at CERN, PPE Division, 1211 Geneva 23, Switzerland.

${ }^{22}$ Now at University of Chicago, Chicago, IL 60637, U.S.A.

${ }^{23}$ Now at TRIUMF, Vancouver, B.C., Canada.

${ }^{24}$ Also at Università di Napoli, Dipartimento di Scienze Fisiche, Napoli, Italy.

${ }^{25}$ Supported by the US Department of Energy, grant DE-FG03-92ER40689.

${ }^{26}$ Now at Università di Pavia, Pavia, Italy.

${ }^{27}$ Now at Max-Planck-Institut f. Kernphysik, Heidelberg, Germany.

${ }^{28}$ Now at Rutgers University, Piscataway, NJ 08854, USA.

${ }^{29}$ Now at Oxford University, Oxford OX1 3RH, U.K.

${ }^{30}$ Partially supported by Colciencias, Colombia.

${ }^{31}$ Now at SSCL, Dallas 75237-3946, TX, U.S.A.

${ }^{32}$ Also at Istituto di Matematica e Fisica, Università di Sassari, Sassari, Italy.
} 



\section{Introduction}

In the minimal standard model, the spontaneous breaking of $S U(2)_{L} \times U(1)_{Y}$ is achieved at the expense of the introduction of a doublet of complex scalar fields $\phi$ in self-interaction. As $\phi$ develops a vacuum expectation value, the $\mathrm{W}$ and $\mathrm{Z}$ bosons acquire their masses while three of the four initial degrees of freedom are absorbed. A single neutral scalar particle, the Higgs boson $H$, therefore results with an unspecified mass $m_{\mathrm{H}}$. However, for a given mass of the Higgs boson, the theory predicts its production rates and partial decay widths unambiguously [1].

At LEP, the bremsstrahlung process $\mathrm{e}^{+} \mathrm{e}^{-} \rightarrow \mathrm{HZ}^{*} \rightarrow$ Hff is the dominant Higgs boson production mechanism. This process has already been investigated by ALEPH [2] and by the other LEP collaborations [3]. With the data collected in 1989 and 1990 (corresponding to about 190,000 hadronic Z decays) ALEPH reported a $95 \%$ C.L. lower limit of $48 \mathrm{GeV} / \mathrm{c}^{2}$ for the Higgs boson mass [2], by searching for almost all the possible topologies arising from the various final states produced in the bremsstrahlung process, depending on the $\mathrm{Z}^{*}$ and $\mathrm{H}$ decay modes.

With the much larger data sample, corresponding to about 1,233,000 hadronic Z decays, collected by ALEPH between 1989 and 1992, the previous analysis becomes sensitive to a higher Higgs boson mass and could naively be repeated without any changes. However, a more complete background study, carried out with accordingly enlarged Monte Carlo samples, led to the following conclusions.

(i) In order to keep the predicted background at a manageable level, some of the selection criteria have to be tightened. After a preselection identical to that of ALEPH's previous analysis, several critical cuts have been optimized on Monte Carlo with the aim of minimizing, on average, the $95 \%$ C.L. upper limit on the number of signal events produced. Since this upper limit depends on the absolute background level, these cuts are expected to change again as more integrated luminosity becomes available.

(ii) After applying the same optimization criterion, only the search for acoplanar jets, relevant for $\mathrm{H} \rightarrow$ hadrons and $\mathrm{Z}^{*} \rightarrow \nu \bar{\nu}$ (the $\mathrm{H} \nu \bar{\nu}$ channel), and the search for energetic lepton pairs in hadronic events, relevant for $\mathrm{H} \rightarrow$ hadrons and $\mathrm{Z}^{*} \rightarrow \mathrm{e}^{+} \mathrm{e}^{-}$or $\mu^{+} \mu^{-}$ (the $\mathrm{Hl}^{+} 1^{-}$channel) are kept in the analysis. The other topological searches, with much smaller relative efficiencies for the Higgs boson search, have to be forsaken because of too small signal-to-background ratios.

These important statistical issues, relevant for the search strategy, are addressed in Section 2. In Sections 3 and 4 the background studies for the searches for energetic lepton pairs in hadronic events and for acoplanar jets are presented in detail. The final result is derived in Section 5. 
A detailed description of the ALEPH detector can be found in Ref. 4. Charged particle momenta are measured by a magnetic spectrometer consisting of a precision vertex detector (VDET) operational since 1991, of a cylindrical drift chamber (ITC), and of a large time projection chamber (TPC). The $1.5 \mathrm{~T}$ axial magnetic field is provided by a superconducting solenoidal coil. The transverse momentum resolution achieved is $\delta p / p=6.10^{-4} p(p$ in $\mathrm{GeV} / c)$.

Photons are measured with an energy resolution $\delta E / E=0.18 / \sqrt{E}+0.01(E$ in $\mathrm{GeV})$ in the electromagnetic calorimeter (ECAL), a 22 radiation length thick sandwich of lead planes and proportional wire chambers with fine longitudinal and transverse read-out segmentation. Photons and electrons are identified in ECAL by the characteristic longitudinal and transverse developments of their associated showers. The $1.5 \mathrm{~m}$ thick iron return yoke of the magnet is interleaved with 23 layers of streamer tubes and acts as a hadronic calorimeter (HCAL) providing, together with ECAL, a measurement of the hadronic energy with a resolution $\delta E / E=0.80 / \sqrt{E}(E$ in $\mathrm{GeV})$ and, together with two layers of muon chambers, muon identification. ECAL is supplemented down to $2^{\circ}$ of the beam axis with an additional electromagnetic calorimeter (LCAL) also used for the luminosity measurement.

An algorithm [2] combines all these measurements to provide a determination of the energy flow, with an accuracy on the measurable total energy $\delta E / E=0.65 / \sqrt{E}(E$ in $\mathrm{GeV})$ and with a typical resolution of $30 \mathrm{mrad}$ on jet direction measurements.

In the data sample used for the analysis reported here, all major components of the detector were required to be simultaneously operational and all major trigger logic relevant to hadronic $\mathrm{Z}$ decays had to be enabled.

\section{Statistical issues}

A search for rare events always faces the two conflicting aims of reaching the highest acceptance for the signal while maintaining the expected background contamination at the lowest level. Some biases may occur in this process when cuts are suggested by an examination of the data. In order to avoid such analysis biases, the sensitivity of the Higgs boson search has been optimized, for $m_{\mathrm{H}}=60 \mathrm{GeV} / c^{2}$, in the following way.

(i) Monte Carlo samples corresponding to typically five times the total integrated luminosity are produced for all known background processes. Signal Monte Carlo samples are also produced.

(ii) A set of variables aimed at discriminating the signal and the various backgrounds is chosen. The cut to be performed on each of these variables is determined by minimizing the average value $\overline{\mathrm{N}}(x)$ of the $95 \%$ C.L. upper limit on the number of signal events produced which would be obtained with an infinity of gedanken experiments in the absence of any signal contribution:

$$
\overline{\mathrm{N}}(x)=\frac{e^{-b(x)}}{\varepsilon(x)}\left\{3.00+4.74 b(x)+6.30 \frac{b^{2}(x)}{2 !}+7.75 \frac{b^{3}(x)}{3 !}+\ldots\right\} .
$$


In this formula, $x$ is the location of the cut and $b(x)$ is the number of background events expected when the cut is applied. This number is determined from the background Monte Carlo distribution of the variable $x$ obtained when all other cuts have been applied, smoothed and extrapolated so that an analytical representation of $b(x)$ is available. The numbers $3.00,4.74,6.30, \ldots$ are the $95 \%$ C.L. upper limits on the number of signal events expected if $0,1,2, \ldots$ background events are observed, while the coefficients of these numbers are the corresponding Poisson probabilities. Finally, $\varepsilon(x)$ is the acceptance of the search for the signal, as a function of the cut location $x$.

An increase of the acceptance $\varepsilon(x)$ of a given topological search leads to a reduction of $\bar{N}(x)$ in the absence of background. Since this also usually increases the amount $b(x)$ of background expected, and hence $\overline{\mathrm{N}}(x)$, a minimization procedure [5] has indeed to be used in order to determine the optimal value of $x$. In practice, this optimum corresponds to a value of $b(x)$ much smaller than one event expected. A complete example of this procedure is given in Section 4. This strategy automatically leads to tighter cuts when the total integrated luminosity increases. Otherwise, while keeping the same acceptance for the signal, the expected background level $b(x)$ would increase, and therefore $\overline{\mathrm{N}}(x)$ too.

The same criterion, i.e. the minimization of $\overline{\mathrm{N}}$, can be used in order to decide a priori which of the various topological searches should be considered in the derivation of the final result, each of those increasing at the same time the overall efficiency and the overall background level. It then turns out that all topological searches except the search for acoplanar jets, relevant for the $\mathrm{H} \nu \bar{\nu}$ channel, have to be abandoned [6].

However, when an event is selected in a topological search such that information on the mass $m_{\mathrm{H}}$ of the candidate Higgs boson is available, it is possible to use this information in a way such that the occurrence of this event will affect the signal production cross-section upper limit only in a restricted mass range [7]. The total visible mass can be used as an estimate of $m_{\mathrm{H}}$ in the $\mathrm{H} \nu \bar{\nu}$ channel, but the method is most powerful in the $\mathrm{Hl}^{+} 1^{-}$channel because of the superior resolution achieved on $m_{\mathrm{H}}$ when it is measured as the mass of the system recoiling against the lepton pair. When the optimization procedure is modified to take into account not only the level but also the mass distribution of the background, the result is that it is justified to keep the $\mathrm{Hl}^{+} 1^{-}$channel in the analysis.

It is also possible to take into account, for any candidate event, the information that it was selected in a given topological search, with a probability proportional to the efficiency of that search [5]. In this way, when simple event counting is performed, the upper limit on the signal production cross-section is not degraded by including additional searches, provided that their contribution to the overall efficiency is sufficiently small, even if they are heavily background contaminated. However, this is not necessarily true if the mass information is used as indicated above, and a complete optimization has to be performed to decide which channels to include. The result is that, with the amount of data now accumulated, only the $\mathrm{H} \nu \bar{\nu}$ and $\mathrm{HI}^{+} 1^{-}$channels are kept. 
Although background Monte-Carlo simulations have been heavily used in the design of the analysis, no background subtraction procedure is foreseen a priori if candidate events should show up in the end. Therefore, the confidence level attached to the final result will not depend on any assumptions related to the background shape or intensity.

\section{Search for energetic lepton pairs in hadronic events}

This search addresses the configuration $(\mathrm{H} \rightarrow$ hadrons $)\left(\mathrm{Z}^{*} \rightarrow \mathrm{e}^{+} \mathrm{e}^{-}\right.$or $\mu^{+} \mu^{-}$), and largely relies on the excellent lepton identification capabilities of the ALEPH detector.

As a preselection, only events with at least six charged particle tracks coming from the interaction point and carrying more than $10 \%$ of the centre-of-mass energy are considered. Energetic pairs are selected as pairs of oppositely charged particle tracks with individual momenta in excess of $3 \mathrm{GeV} / c$, with a scalar sum of momenta greater than $20 \mathrm{GeV} / c$, and with an invariant mass greater than $5 \mathrm{GeV} / c^{2}$. Furthermore, the scalar sum of their transverse momenta with respect to the thrust axis of the rest of the event has to exceed $15 \mathrm{GeV} / c$. Events with no such pairs are rejected.

For each pair, at least one of the two particles has to be positively identified as an electron or as a muon, according to the strict criteria of Ref. 2. These criteria are also applied to the second particle except when the track extrapolates to a non-instrumented region of the ECAL for electrons, or of the HCAL for muons. The pairs formed with an electron and a muon are rejected.

This preselection, which is identical to the one presented in Ref. 2, has been applied to a Monte Carlo sample of $1,700,000$ hadronic $\mathrm{Z}$ decays, which revealed that:

(i) the requirement that one of the two tracks be isolated is particularly efficient at rejecting background events from $\mathrm{Z} \rightarrow \mathrm{q} \overline{\mathrm{q}}$ while keeping a high efficiency for the signal. Here, a track is isolated when no other charged particles are found inside a cone of $30^{\circ}$ half-opening angle around its momentum direction and when less than $1 \mathrm{GeV}$ of neutral energy - apart from identified bremsstrahlung photons - is detected in the same cone. The size of the isolation cone has been determined with the optimization technique presented in the previous section;

(ii) the bulk of the background events, when a looser isolation cut is applied, comes from $\mathrm{Z}$ decays into $\mathrm{b} \overline{\mathrm{b}}$, especially when the two b-quarks decay semi-leptonically.

Therefore, a Monte Carlo sample of $350,000 \mathrm{Z} \rightarrow \mathrm{b} \overline{\mathrm{b}}$ events corresponding to $1,600,000$ hadronic $\mathrm{Z}$ decays and another sample of $47,000 \mathrm{Z} \rightarrow \mathrm{b} \overline{\mathrm{b}}$ events with two subsequent b-quark semi-leptonic decays corresponding to 4,000,000 hadronic $\mathrm{Z}$ decays, have been simulated and analyzed. No events from any of these samples, equivalent altogether to almost six times the integrated luminosity recorded by now, were found to satisfy the above selection criteria, confirming the low level of expected background. 
At this level, the ultimate background source to the $\mathrm{Hl}^{+} 1^{-}$channel is the four-fermion process $\mathrm{e}^{+} \mathrm{e}^{-} \rightarrow 1^{+} 1^{-} \mathrm{q} \overline{\mathrm{q}}$, characterized by a topology very close to that of the signal. In the electron channel, the cross-section for this process develops a pole when at least one of the two electrons is at very low angle with respect to the beam axis. A luminosity equivalent to five times the data has been simulated for this topology, due to the socalled two-photon processes, using a dedicated Monte Carlo program described in Ref. 8. When both electrons escape undetected along the beam direction, this reaction does not contribute to the topology of interest here. However, a small contribution arises from the configuration in which one of the electrons is scattered at a large angle to the beam direction, with the other one still being undetected, when a real or fake electron is found in the recoiling hadronic system. This contribution is removed by requiring that (i) $q_{e} P_{L}^{\text {mis }}$ be smaller than $15 \%$ of the centre-of-mass energy, where $q_{e}$ is the sign of the charge of the most energetic electron and $P_{L}^{m i s}$ the signed missing longitudinal momentum, and (ii) by rejecting events with more than $10 \mathrm{GeV}$ within $12^{\circ}$ of the beam axis.

One thousand $1^{+} 1^{-} \mathrm{q} \overline{\mathrm{q}}$ events with the two leptons in the detector acceptance, corresponding to about thirty times the actual recorded luminosity, have been generated for each of the three lepton flavours. This has been done using the FERMISV Monte Carlo generator [9] which includes all the lowest order diagrams contributing to this final state and the first order QED corrections, but no QCD corrections. Since the leptons are required in the preselection to form a rather large invariant mass and to be very energetic, the recoiling hadronic system tends to be characterized by a low mass and a low multiplicity. For the $\mathrm{e}^{+} \mathrm{e}^{-} \mathrm{q} \overline{\mathrm{q}}$ and the $\mu^{+} \mu^{-} \mathrm{q} \overline{\mathrm{q}}$ final states, the hadronic mass can be accurately computed from the lepton momenta, using energy-momentum conservation. This recoil mass is required to be larger than $40 \mathrm{GeV} / c^{2}$. To remove most of the events coming from the $\tau^{+} \tau^{-} \mathrm{q}_{\bar{q}}$ final state for which this technique cannot be used, it is required that the total charged multiplicity be at least 8 when the missing energy is larger than $10 \%$ of the centre-of-mass energy.

Altogether, FERMISV predicts $3.1 \pm 0.3$ background events while no events are observed in the data. The distribution of the recoil mass, presented in Fig. 1 after the removal of the $40 \mathrm{GeV} / c^{2}$ cut, exhibits a slight excess at low values and a deficit at high values with respect to the absolutely normalized prediction, but these discrepancies are alleviated by including QCD corrections. For hadronic masses below $20 \mathrm{GeV} / c^{2}$, the diagram of Fig. $2 \mathrm{a}$ is dominant and the cross-section should be increased by $\sim 20 \%$, taking into account the

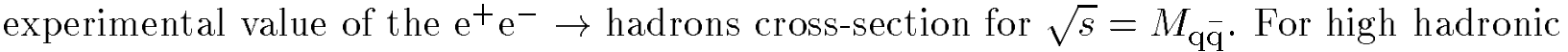
masses, when the diagram of Fig. $2 \mathrm{~b}$ becomes dominant, the parton level calculation is inappropriate since gluon radiation competes with and reduces virtual photon radiation, as has been shown to be the case for real photon emission [10]. With this effect taken into account, the number of events expected to be seen above $40 \mathrm{GeV} / c^{2}$ is reduced by a factor of $\sim 2$, leading to a better agreement between observation and expectation.

The $\sim 1.5$ background events expected are to be compared to 0.67 events expected from a $60 \mathrm{GeV} / c^{2}$ Higgs boson. However, as mentioned in Section 2, the resolution on the recoil mass ( $500 \mathrm{MeV} / c^{2}$ for a $60 \mathrm{GeV} / c^{2}$ Higgs boson) could be used to improve the effective signal-to-background ratio. 


\section{Search for acoplanar jets}

In this section, the topology of interest consists of an acoplanar hadronic system accompanied by missing energy. It addresses the configuration $(\mathrm{H} \rightarrow$ hadrons $)\left(\mathrm{Z}^{*} \rightarrow \nu \bar{\nu}\right)$ and has been optimized for the search for a $60 \mathrm{GeV} / c^{2}$ Higgs boson, making use of the accurate reconstruction of the energy flow in the ALEPH detector.

For this topology, the preselection is performed on events with a total visible mass smaller than $70 \mathrm{GeV} / \mathrm{c}^{2}$ and with at least eight charged particle tracks coming from the interaction point and carrying more than $10 \%$ of the centre-of-mass energy. Again, this preselection is identical to the one presented in Ref. 2, and is only briefly recalled here.

In order to avoid energy losses around the beam direction, the fraction of the total visible energy that is measured beyond $30^{\circ}$ of that direction is required to exceed $60 \%$, and the energy measured within $12^{\circ}$ of the beam axis has to be smaller than $3 \mathrm{GeV}$. The events are then divided into two hemispheres by a plane perpendicular to the thrust axis, and the angle between the directions of the total momenta measured in the two hemispheres (the acollinearity angle) is required to be smaller than $165^{\circ}$, which removes the bulk of the $\mathrm{Z}$ decays into two back-to-back jets.

Events resulting from photon-photon collisions are usually not collinear and exhibit a large missing energy along the beam axis due to the initial electron and positron which escape detection. They are rejected by requiring a visible mass above $25 \mathrm{GeV} / c^{2}$ when the total momentum transverse to the beam is smaller than $10 \%$ of the centre-of-mass energy. In addition, the invariant masses measured in both hemispheres are required to be larger than $2.5 \mathrm{GeV} / c^{2}$. This last cut is also very efficient against the few remaining $\mathrm{Z}$ decays into $\tau^{+} \tau^{-}$and against the $\tau^{+} \tau^{-} q \bar{q}$ four-fermion final state with a low mass $q \bar{q}$ pair.

A large acollinearity might also be due to an $\mathrm{e}^{+} \mathrm{e}^{-}$annihilation into two jets accompanied by a hard initial state radiation. To remove such events, it is required that the angle between the total momentum direction and the beam axis be larger than $21.8^{\circ}$, and that the angle between the two hemisphere directions projected onto a plane perpendicular to the beam (the acoplanarity angle) be smaller than $175^{\circ}$.

Although this preselection is aimed at rejecting two-jet events, it is also efficient against three-jet events, when the three jet energies are well measured. However, when at least one of the energies is mismeasured, the directions of the total momentum and of the two hemisphere momenta are affected so that the distributions of the acollinearity and acoplanarity angles are smeared out. Three configurations can be distinguished.

(i) When only one jet energy is mismeasured, the total missing momentum clearly points towards the direction of this jet, and is therefore not isolated. This type of events is rejected by requiring that less than $1 \mathrm{GeV}$ be detected in a cone of half-opening angle $25.8^{\circ}$ around the missing momentum direction. This cut is also efficient at removing the $\tau^{+} \tau^{-} \mathrm{q} \overline{\mathrm{q}}$ four-fermion final state with a low-mass $\tau^{+} \tau^{-}$pair. 
(ii) When at least two jet energies are poorly measured, the previous cut is not efficient because the direction of the missing momentum is no longer related to a given jet. However, the three jet directions tend to remain in a given plane, which can be characterized by a value close to $360^{\circ}$ for the sum $\mathcal{S}$ of the three jet-jet angles obtained when the event is forced to form three jets. The distributions of $\mathcal{S}$ obtained from the 1,700,000 hadronic $\mathrm{Z}$ decay Monte Carlo sample and from a sample of 7,000 $\mathrm{e}^{+} \mathrm{e}^{-} \rightarrow \mathrm{H} \nu \bar{\nu}$ simulated events with $m_{\mathrm{H}}=60 \mathrm{GeV} / c^{2}$ are presented in Fig. 3. Only events satisfying all the above selection criteria, together with the one described below, enter these distributions. After applying the optimization procedure, $\mathcal{S}$ is required to be smaller than $345^{\circ}$.

(iii) The remaining background events are also three-jet events with at least one jet energy mismeasured, but now accompanied by a hard initial state radiation. The simple properties (i) and (ii) are therefore lost, but this situation can still be addressed using energy-momentum conservation since it is possible to recompute the energies of the three jets assuming that their directions have been well measured and that a photon escaped along the beam axis. The energy of the undetected photon is also computed by this method. The topology of such background events is sketched in Fig. 4a, and that of the events expected from the signal in Fig. 4b. All of the recomputed energies should be positive in the first case. In contrast, for the signal, since the jets coming from a Higgs boson decay tend to be contained in the hemisphere defined by the original Higgs direction, one of the recomputed jet energies has to be negative in order to fake a jet in the opposite hemisphere.

In order to cope with this topology, the minimum $E_{\min }$ of the three recomputed jet energies is used. The distribution of the acoplanarity angle $v s E_{\min }$ is shown in Fig. 5a for background events. It is obtained from the 1,700,000 hadronic $\mathrm{Z}$ decay Monte Carlo sample enriched by a sample of $6,000 q \bar{q} \gamma$ simulated events with a radiated energy in excess of $10 \mathrm{GeV}$, corresponding altogether to $6,000,000$ hadronic $\mathrm{Z}$ decays. All these events indeed tend to have a positive $E_{\min }$ while preserving an acoplanarity angle close to $180^{\circ}$, in contrast to the events from $\mathrm{e}^{+} \mathrm{e}^{-} \rightarrow \mathrm{H} \nu \bar{\nu}$, as shown in Fig. $5 \mathrm{c}$. All the Monte Carlo background events, corresponding to $2.8 \pm 0.7$ events expected, are rejected by the requirement that the acoplanarity angle be smaller than $162^{\circ}$ when $E_{\text {min }}$ exceeds $-1 \mathrm{GeV}$. As shown in Fig. 5b, the 2 events remaining in the data are also removed by these cuts.

The value of the acoplanarity cut has been set with the optimization procedure described in Section 2. First, the distribution of the acoplanarity angle is determined by fitting the Monte Carlo distribution, inevitably statistically limited after all other cuts have been applied, with an exponential shape. The result of the fit, shown in Fig. 5d, has been checked to reproduce accurately the number of events observed in the tail of the Monte Carlo distribution. The efficiency of the selection is then obtained from the $\mathrm{H} \nu \bar{\nu}$ Monte Carlo sample with $m_{\mathrm{H}}=60 \mathrm{GeV} / c^{2}$ and is shown in Fig. 5e as a function of the value of the acoplanarity cut. The optimized value is finally computed by minimizing, when the acoplanarity cut is varied, the average of the $95 \%$ C.L. upper limit on the number of signal events produced. As shown in Fig. 5f, the absolute minimum is reached when 
the acoplanarity cut is set at $162^{\circ}$. With an integrated luminosity twice as large as the luminosity recorded by now, and provided that the other criteria remain unchanged, the cut would be set at $160^{\circ}$.

In order to understand the origin of the background events rejected only by the cuts discussed in (i) and (ii) once this last criterion is applied, the isolation cut has been loosened to $3 \mathrm{GeV}$ and the $\mathcal{S}$ cut to $355^{\circ}$. It is then observed that most of the background events satisfying these looser criteria come from $\mathrm{Z}$ decays into $\mathrm{b} \bar{b}$, especially when at least one of the two b-quarks decays semi-leptonically. The Monte Carlo samples of 350,000 $\mathrm{Z} \rightarrow \mathrm{b} \overline{\mathrm{b}}$ events corresponding to $1,600,000$ hadronic $\mathrm{Z}$ decays, and of $47,000 \mathrm{Z} \rightarrow \mathrm{b} \overline{\mathrm{b}}$ events with two subsequent semi-leptonic decays, corresponding to 4,000,000 hadronic $\mathrm{Z}$ decays, can therefore be used to check the robustness of these two cuts. No events from the first sample survive the cuts. One event of the second sample combining two semi-leptonic decays, a clean three-jet topology and a radiated photon energy of $20 \mathrm{GeV}$ along the beam direction, is kept with an $\mathcal{S}$ value of $341^{\circ}$.

This corresponds to 0.18 events expected in the ALEPH data from $\mathrm{Z}$ decays into hadrons. Less than 0.20 events are expected from $\mathrm{Z}$ decays into $\tau^{+} \tau^{-}$or from two-photon processes, and 0.06 events are expected from the four-fermion process $\mathrm{e}^{+} \mathrm{e}^{-} \rightarrow \tau^{+} \tau^{-} \mathrm{q} \overline{\mathrm{q}}$. Altogether, a total of $0.24_{-0.15}^{+0.27}$ background events are expected to be found, to be compared to 1.54 signal events for a $60 \mathrm{GeV} / c^{2}$ mass Higgs boson. Even when the initial $70 \mathrm{GeV} / \mathrm{c}^{2}$ mass cut is removed, no additional background events are expected and no events are observed in the data.

As an alternative to the cut optimization method described in Section 2, a neural network technique has been developed for the $\mathrm{H} \nu \bar{\nu}$ channel. In this method, a feed-forward neural network [11] with three twelve-node hidden layers is trained on various Monte Carlo samples including (i) 200,000 hadronic Z decays, (ii) several dedicated samples for specific backgrounds and (iii) the $\mathrm{H} \nu \bar{\nu}$ signal. From eight input variables essentially identical to those described above, a discriminating output variable is produced on which a cut is made. In the case of a single variable, placing the cut at the edge of the last event seen in the data does not cause any bias. With such a cut on the output variable, the efficiency is $36 \%$ for $m_{\mathrm{H}}=55 \mathrm{GeV} / c^{2}$, which is sizeably smaller than the $51 \%$ of the previous method. Since it has not yet been possible to achieve a higher efficiency in this first neural network approach, the results reported in this letter are derived using the classical cut technique.

\section{Result of the analysis}

The two topological searches used in this analysis specifically address the $\mathrm{Hl}^{+} 1^{-}$and the $\mathrm{H} \nu \bar{\nu}$ channels, but are also sensitive, although with a low efficiency, to the channels $(\mathrm{H} \rightarrow$ hadrons $)\left(\mathrm{Z}^{*} \rightarrow \tau^{+} \tau^{-}\right)$and $\left(\mathrm{H} \rightarrow \tau^{+} \tau^{-}\right)$( $\mathrm{Z}^{*} \rightarrow$ hadrons $)$.

No events were observed in a data sample corresponding to about 1,233,000 hadronic $\mathrm{Z}$ decays. The numbers of signal events expected in this sample are shown in Table 1 for different Higgs boson mass hypotheses. The production cross-section has been determined 
as in Ref. 2, except that the radiative corrections are now taken into account as calculated in Ref. 12. Furthermore, the numbers of Table 1 have been derived with a branching ratio of the Higgs boson into $\tau^{+} \tau^{-}$of $\sim 8 \%$, as obtained [13] with a calculation of the hadronic partial widths up to the third order of QCD and using a b-quark constituent mass of $4.7 \pm 0.3 \mathrm{GeV} / c^{2}$. The various sources of systematic uncertainties on these numbers can be listed as follows.

- An uncertainty of $0.2 \%$ is due to the determination of the number of multihadronic events in the data sample.

- The allowed range for the top-quark mass, $m_{\mathrm{t}}=134_{-25}^{+22} \mathrm{GeV} / \mathrm{c}^{2}$, obtained from ALEPH data [14] with $m_{\mathrm{H}}=60 \mathrm{GeV} / c^{2}$, results in an uncertainty of $\pm 0.6 \%$ on the ratio of the $\mathrm{HZ}^{*}$ to $\mathrm{q} \overline{\mathrm{q}}$ cross-sections.

- The limited signal Monte-Carlo statistics introduces a contribution of $0.9 \%$ to the uncertainty.

- The uncertainties in the hadronization and fragmentation mechanism of the q $\bar{q}$ pair originating from the Higgs boson contribute another $1 \%$ for the $\mathrm{H} \nu \bar{\nu}$ channel. The $\mathrm{Hl}^{+} 1^{-}$channel is much less affected by these uncertainties since the corresponding selection criteria are essentially related to the leptons.

- The modelling of the lepton identification is accurate at the $2 \%$ level (this affects only the $\mathrm{Hl}^{+} 1^{-}$channel).

- Finally, the ambiguity on the value of the b-quark constituent mass entering the calculation of the $(\mathrm{H} \rightarrow \mathrm{b} \bar{b})$ decay partial width introduces a $\pm 1 \%$ uncertainty on the corresponding branching ratio. This translates into $\pm 0.7 \%$ for the number of events expected.

The overall systematic uncertainty is therefore below $2 \%$. The number of signal events expected, conservatively reduced by this amount, is presented in Fig. 6 as a function of the Higgs boson mass. With no events observed, an improved 95\% C.L. Higgs boson mass lower limit of $58.4 \mathrm{GeV} / c^{2}$ is obtained.

\section{Acknowledgements}

We wish to thank our colleagues from the accelerator divisions for the successful operation of LEP. We are indebted to the engineers and technicians in all our institutions for their contribution to the excellent performance of ALEPH. Those of us from nonmember countries thank CERN for its hospitality. 


\section{References}

1. J.F. Gunion, H.E. Haber, G. Kane. and S. Dawson, "The Higgs Hunter's Guide", Ed. Addison-Wesley (1990);

P.J. Franzini et al., "Z Physics at LEP", eds G. Altarelli, R. Kleiss and C. Verzegnassi, CERN 89-08 (1989).

2. D. Decamp et al., (ALEPH Coll.), Phys. Rep. 216 (1992), 253.

3. P. Abreu et al., (DELPHI Coll.), Nucl. Phys. B273 (1992), 3;

O. Adriani et al., (L3 Coll.), Phys. Lett. 303B (1993), 391;

M.Z. Akrawy et al., (OPAL Coll.), Phys. Lett. 253B (1991), 511.

4. D. Decamp et al., (ALEPH Coll.), Nucl. Instrum. Methods A 294 (1990), 121.

5. J.-F. Grivaz and F. Le Diberder, "Complementary analyses and acceptance optimization in new particle searches", LAL 92-37, June 1992.

6. D. Decamp et al., (ALEPH Coll.), "Improved mass limit for the standard model Higgs boson", contributed paper to the XXVIth Int. Conf. on High Energy Physics, Dallas, August 1992.

7. J.-F. Grivaz and F. Le Diberder, "On the determination of a mass lower limit for the Higgs boson in the presence of candidate events", LAL 92-23, April 1992, and Erratum, LAL 92-45, August 1992, to be published in Nucl. Instrum. Methods.

8. P.H. Daverveldt, Comput. Phys. Commun. 40 (1986) 285.

9. J.M. Hilgart, R. Kleiss and F. Le Diberder, Comput. Phys. Commun. 75 (1993), 191.

10. D. Decamp et al., (ALEPH Coll.), Phys. Lett. 264B (1991), 476.

11. J. Hertz, A. Krogh and R.G. Palmer, "Introduction to the theory of neural computation", Addison-Wesley Publishing Company.

12. B.A. Kniehl, Phys. Lett. 282B (1992), 249.

13. A. Djouadi, J. Kalinowski and P.M. Zerwas, Z. Phys. C57 (1993), 569;

Z. Kunszt and F. Zwirner, "Testing the Higgs sector of the MSSM at large hadron colliders", CERN-TH 6150/91, December 1991.

14. D. Buskulic et al., (ALEPH Coll.), "Update of electroweak parameters from Z decays", CERN-PPE/93-40 (March 1993), submitted to Z. Phys. C. 


\begin{tabular}{|c|c|c|c|c|c|c|c|c|}
\hline Channel & \multicolumn{2}{|c|}{$\mathrm{H} \nu \bar{\nu}$} & \multicolumn{2}{c|}{$\mathrm{He}^{+} \mathrm{e}^{-}$} & \multicolumn{2}{c|}{$\mathrm{H} \mu^{+} \mu^{-}$} & Other & Total \\
\hline$m_{\mathrm{H}}\left(\mathrm{GeV} / c^{2}\right)$ & Eff. (\%) & $N_{\text {exp }}$ & Eff. (\%) & $N_{\exp }$ & Eff. $(\%)$ & $N_{\exp }$ & $N_{\exp }$ & $N_{\exp }$ \\
\hline 50 & 55 & 7.68 & 59 & 1.39 & 69 & 1.63 & 0.41 & 11.11 \\
\hline 55 & 51 & 3.74 & 55 & 0.69 & 66 & 0.84 & 0.19 & 5.46 \\
\hline 60 & 43 & 1.54 & 49 & 0.30 & 61 & 0.37 & 0.07 & 2.28 \\
\hline 65 & 34 & 0.53 & 44 & 0.12 & 56 & 0.15 & 0.02 & 0.82 \\
\hline
\end{tabular}

Table 1. Efficiencies and numbers of signal events expected in the searches for acoplanar jets and for energetic lepton pairs in hadronic events, when applied to the $\mathrm{H} \nu \bar{\nu}, \mathrm{He}^{+} \mathrm{e}^{-}$, $\mathrm{H} \mu^{+} \mu^{-}$channels, with $\mathrm{H} \rightarrow$ hadrons, and to all the other possible final states, such as $\tau^{+} \tau^{-} \mathrm{q} \overline{\mathrm{q}}$, for Higgs boson masses of $50,55,60$ and $65 \mathrm{GeV} / c^{2}$. 


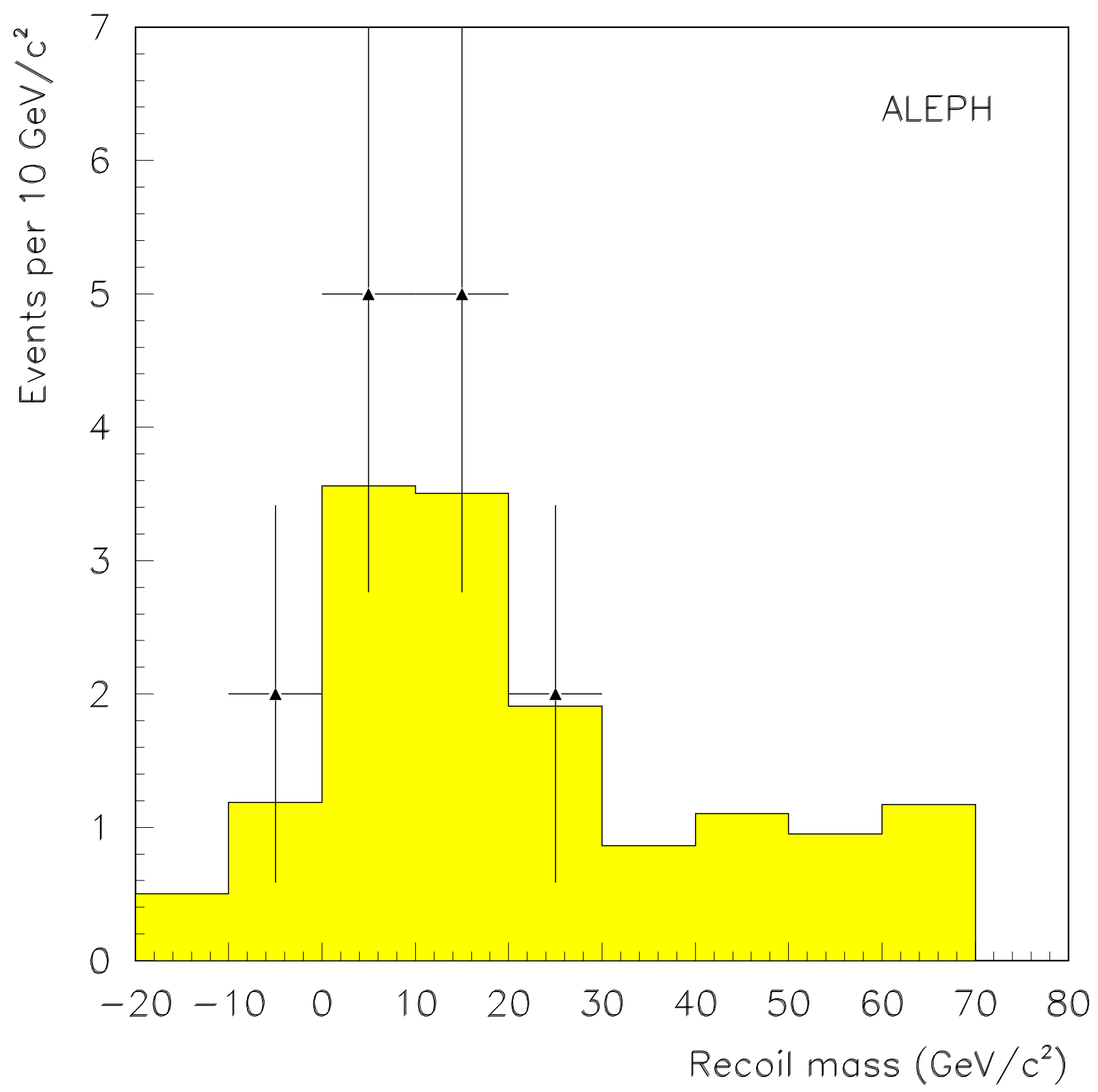

Figure 1: Distribution of the mass recoiling against the lepton pair, when relaxing the cut at $40 \mathrm{GeV} / c^{2}$ on this quantity, for $\mathrm{e}^{+} \mathrm{e}^{-} \rightarrow 1^{+} 1^{-} \mathrm{q} \overline{\mathrm{q}}$ Monte-Carlo events generated with the FERMISV program with an absolute normalization (shadowed histogram), and for the data (points with error bars). Negative squared recoil masses $m_{r e c}^{2}$ occur when the sum of the measured lepton momenta is larger than the centre-of-mass energy; in this case, the recoil mass is defined as $-\sqrt{\left|m_{r e c}^{2}\right|}$. 


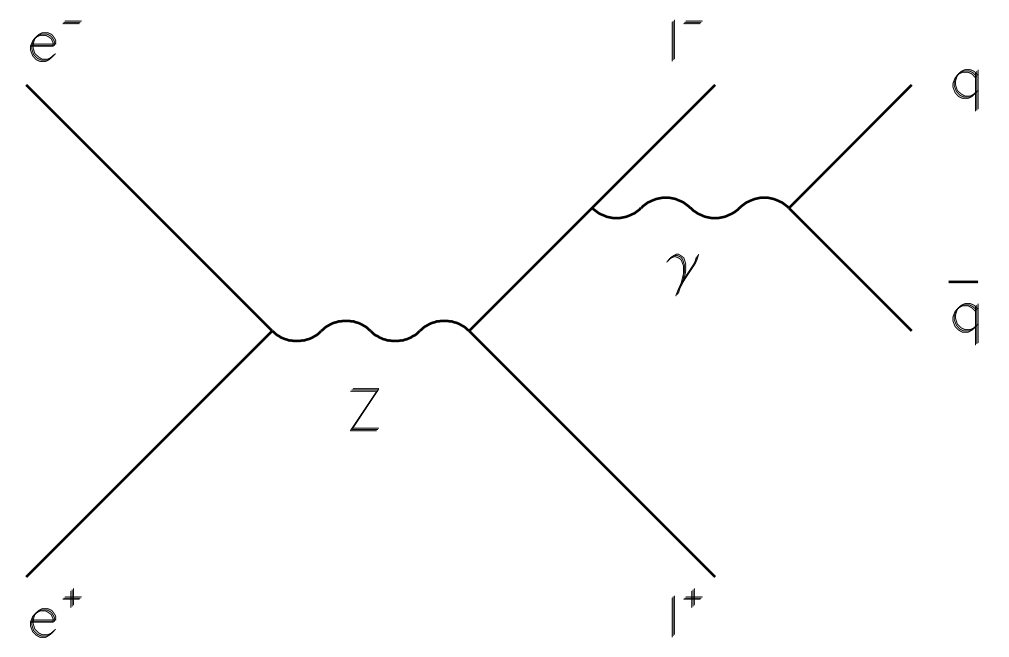

(a)

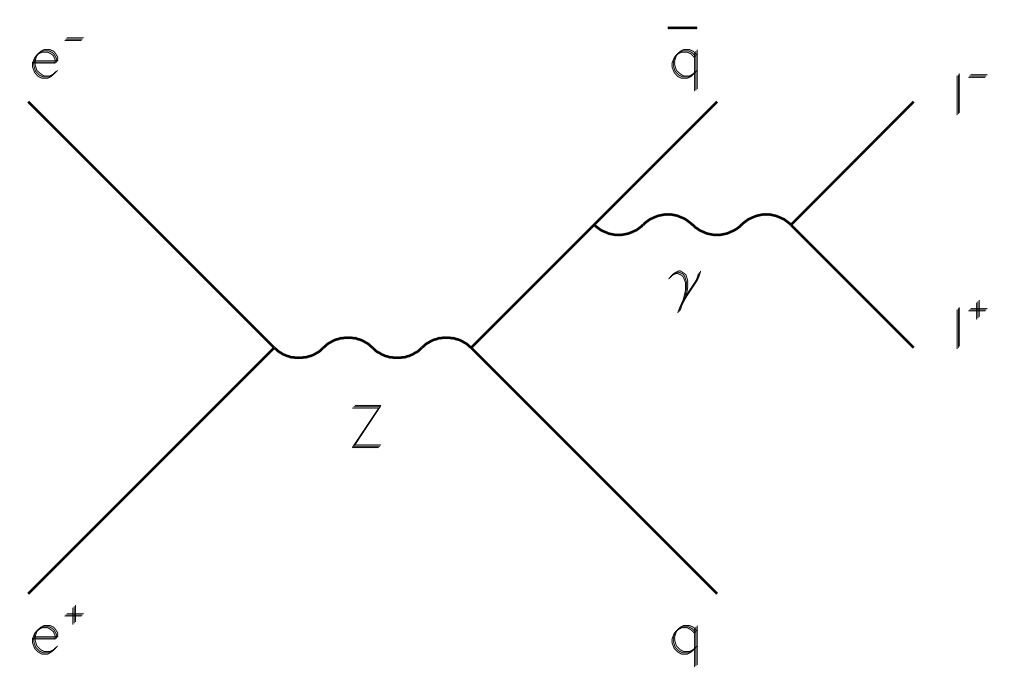

(b)

Figure 2: Two Feynman graphs for the process $\mathrm{e}^{+} \mathrm{e}^{-} \rightarrow 1^{+} 1^{-} \mathrm{q} \overline{\mathrm{q}}$ :

a) contributing essentially to the low recoil mass domain;

b) contributing essentially to the high recoil mass domain. 

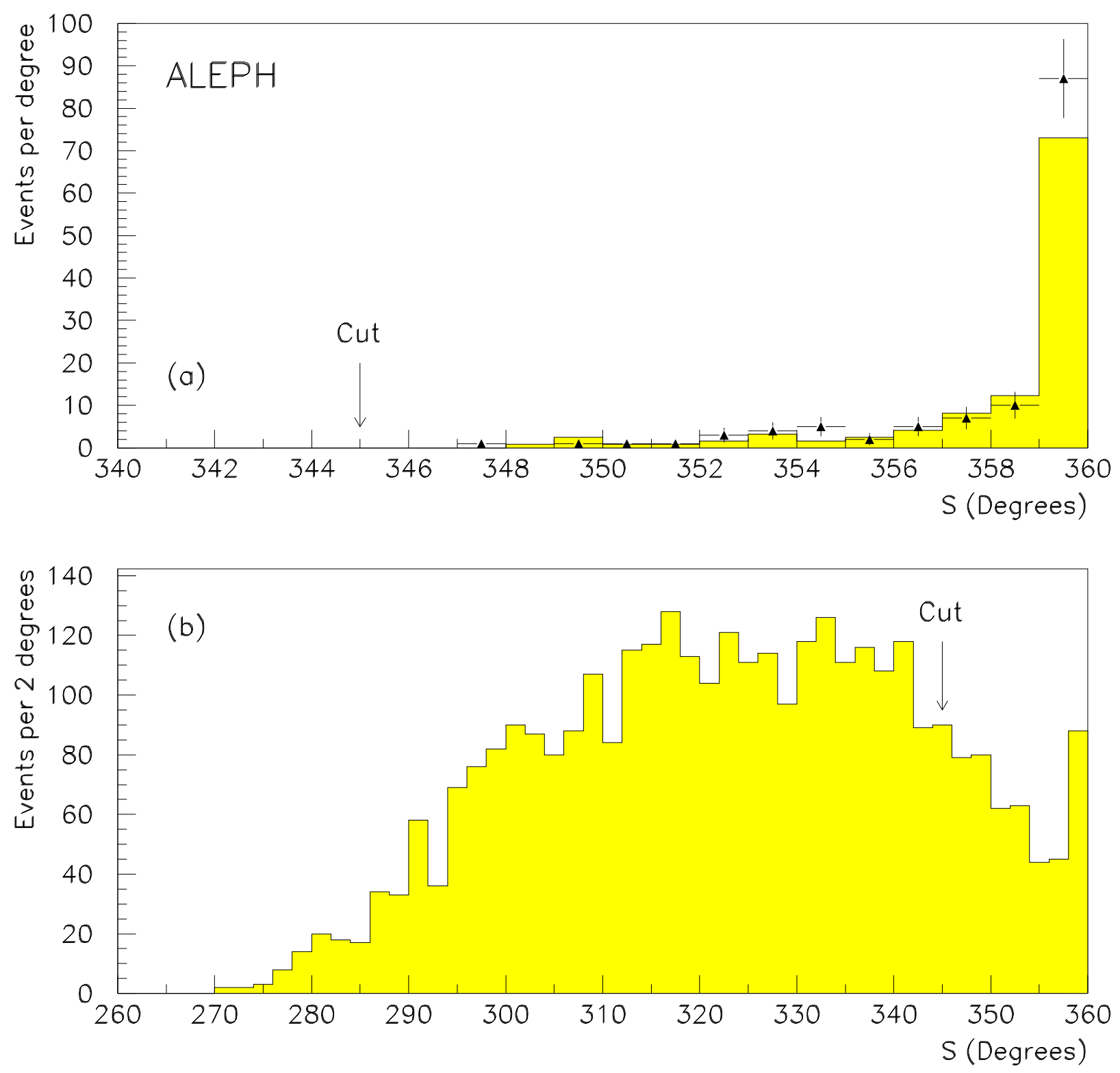

Figure 3: Distribution of the sum S (see text), all other cuts being applied:

a) for the 1,700,000 hadronic $Z$ decay Monte Carlo sample with an absolute normalization (shadowed histogram) and for the data (triangles with error bars);

b) for $\mathrm{H} \nu \bar{\nu}$ simulated events with $m_{\mathrm{H}}=60 \mathrm{GeV} / c^{2}$.

Note the difference of scale between the two figures. The cut at $345^{\circ}$ is indicated by an arrow. 


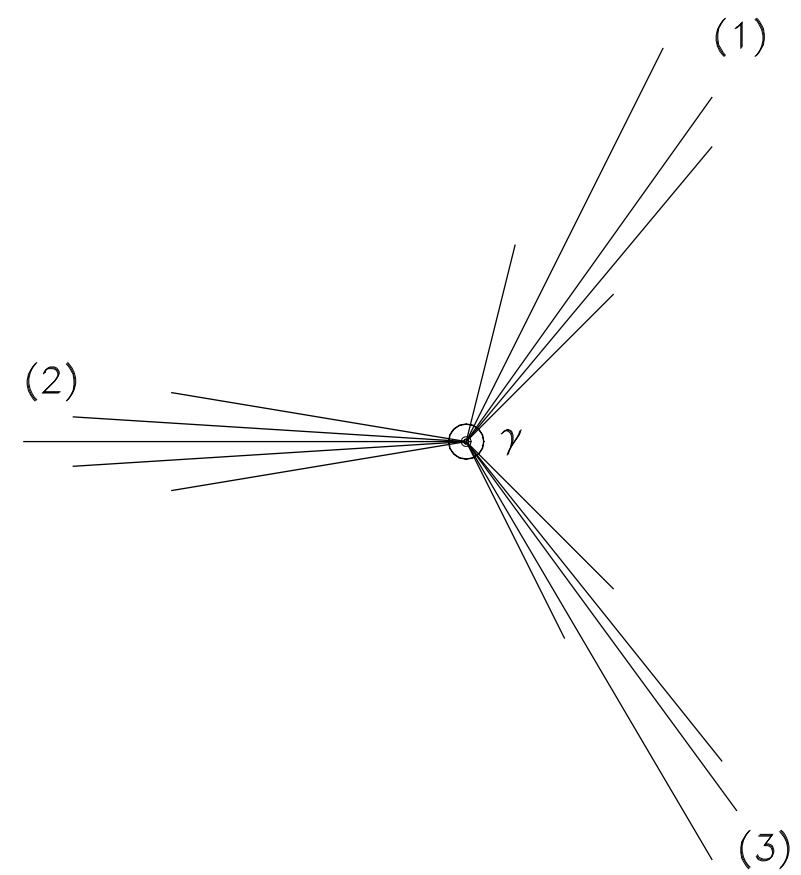

(a)

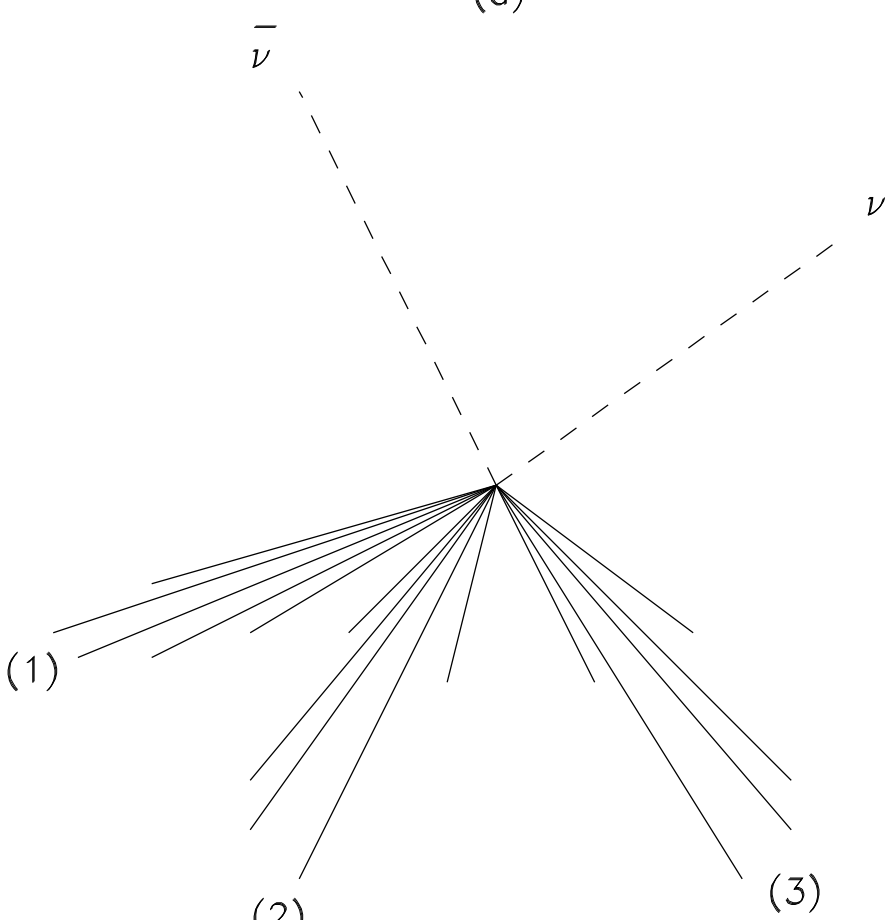

(b)

Figure 4: Schematic view of the topology of final states, seen in the plane transverse to the beam axis:

a) for a $q \bar{q} g \gamma$ event, all the recomputed jet energies are positive;

b) for a $\mathrm{H} \nu \bar{\nu}$ event, the recomputed energy of jet 2 is negative. 

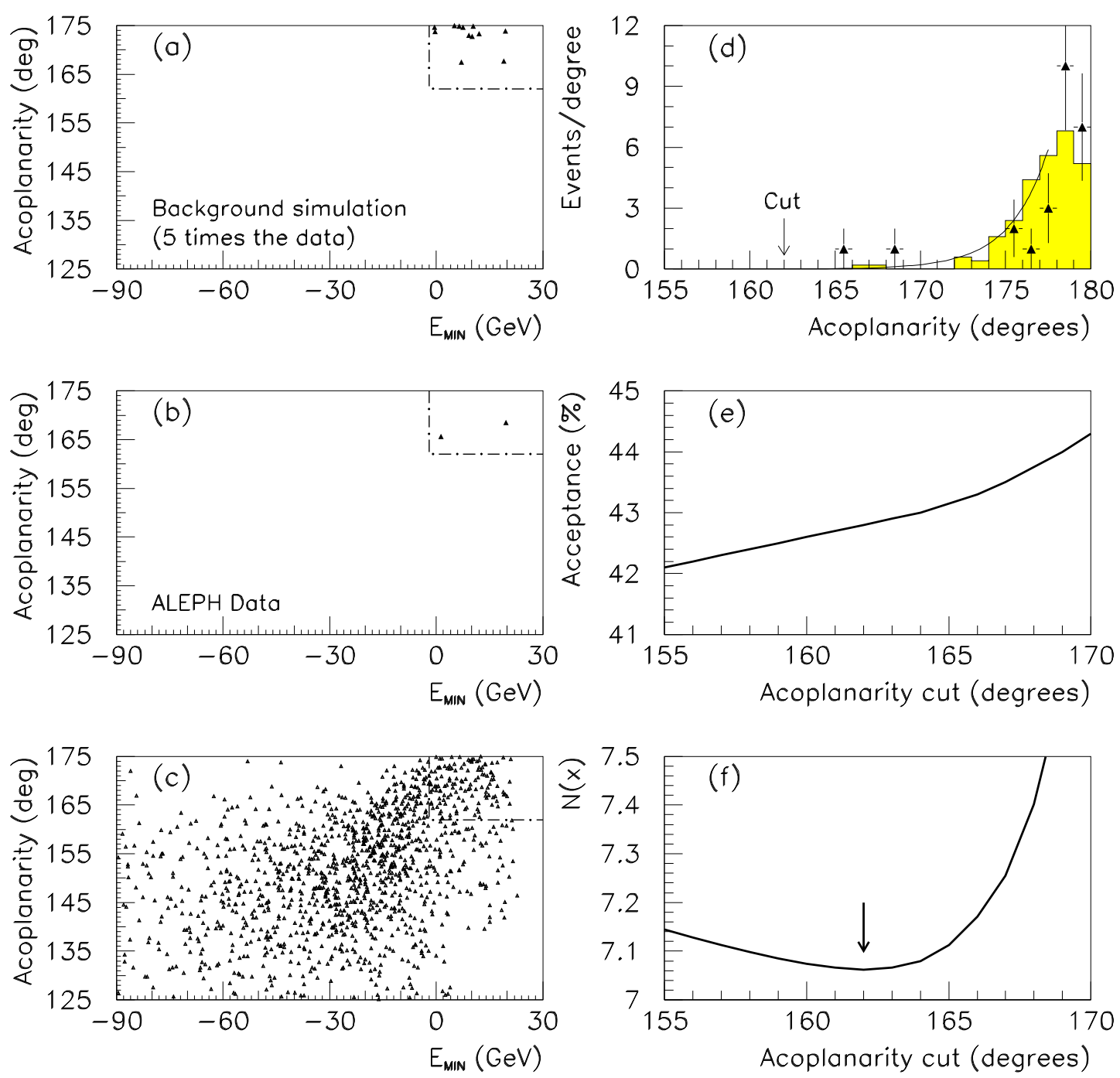

Figure 5: Distribution of the acoplanarity angle versus the minimum of the three recomputed jet energies (see text), all other cuts being applied:

a) for a Monte Carlo sample equivalent to six million hadronic $Z$ decays;

b) for the data;

c) for $\mathrm{H} \nu \bar{\nu}$ simulated events with $m_{\mathrm{H}}=60 \mathrm{GeV} / c^{2}$.

d) Distribution of the acoplanarity angle, all other cuts being applied, for the data (triangles with error bars) and the Monte Carlo (shadowed histogram). The full line results from a fit of the Monte Carlo distribution with an exponential shape, and is used to determine the number of background events expected.

e) Acceptance of the search as a function of the acoplanarity cut, for a Higgs boson mass of $60 \mathrm{GeV} / c^{2}$.

f) Average of the $95 \%$ C.L. upper limit on the number of $\mathrm{H} \nu \bar{\nu}$ events produced as a function of the acoplanarity cut, for a Higgs boson mass of $60 \mathrm{GeV} / \mathrm{c}^{2}$. The minimum (indicated by an arrow) is reached for a cut at $162^{\circ}$.

The optimized cut is indicated by a dashed-dotted line in (a), (b), and (c), and by an arrow in $(d)$. 


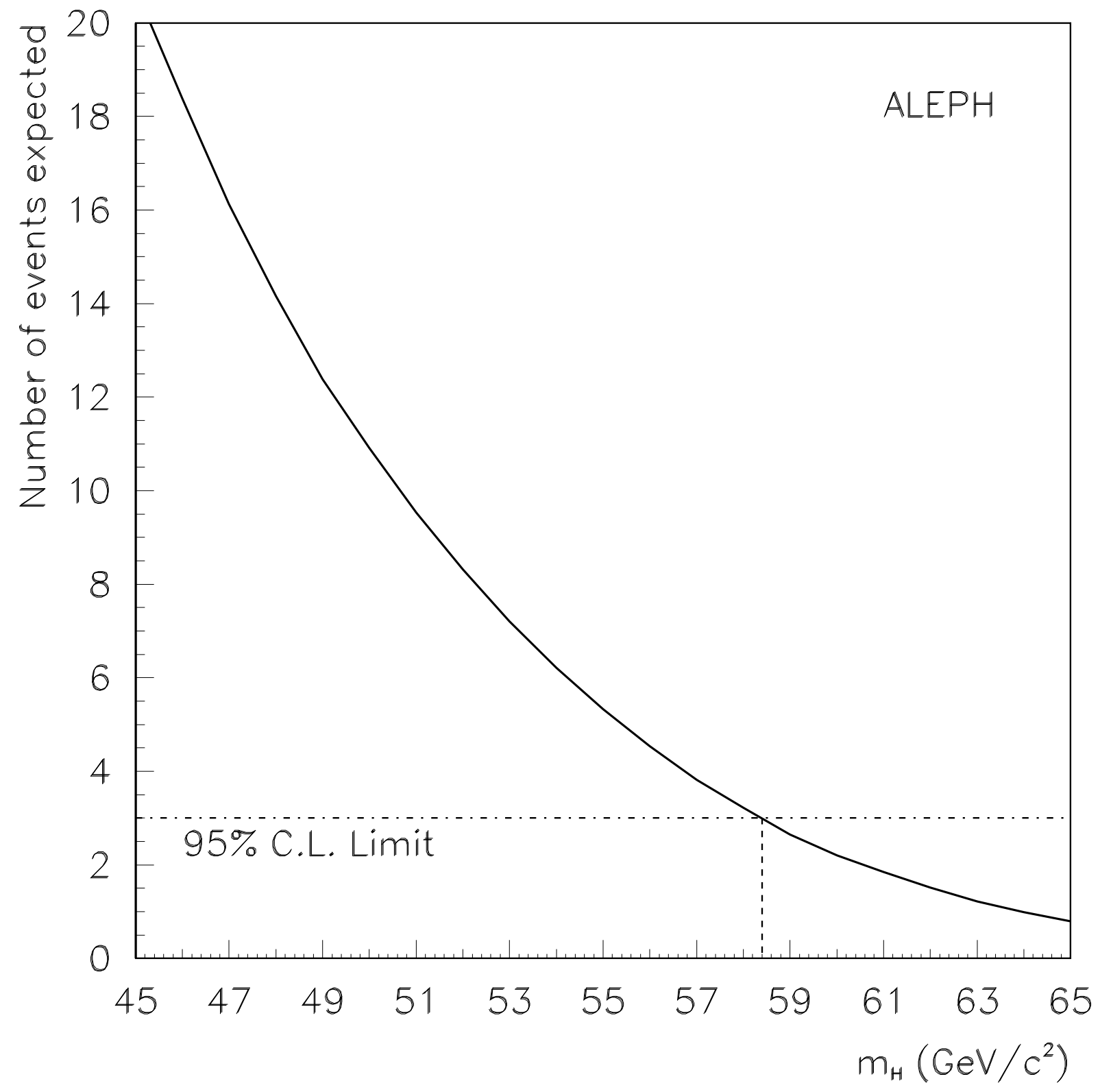

Figure 6: Number of signal events expected, reduced by the overall systematic uncertainty of $2 \%$, as a function of the Higgs boson mass. Also shown is the $95 \%$ C.L. upper limit on the number of signal events. 